\begin{tabular}{c} 
International Journal of Engineering \& Technology, $7(2.13)(2018) 7-12$ \\
International Journal of Engineering \& Technology \\
WPC \\
Website: www.sciencepubco.com/index.php/IJET \\
Research Paper \\
\hline
\end{tabular}

\title{
Free Vibrations of Thin-Walled Box-Section Bars Allowing for Shear Strains
}

\author{
Aleksandr Gavrilov ${ }^{1}$, Grigoriy Grebenyuk ${ }^{2}$, Nikolay Morozov¹, Andrey Grehov ${ }^{1}$ \\ ${ }^{1}$ Orenburg State University, 13 Pobedy avenue, Orenburg, Russia, 460018 \\ ${ }^{2}$ Novosibirsk State University of Architecture and Civil Engineering, 113 Leningradskaya street, Novosibirsk, Russia, 630008 \\ Email: olga.chorosova@mail.ru
}

\begin{abstract}
Purpose. The purpose is to study free vibrations of thin-walled bar with combined bisymmetrical section allowing for the effects of shear strains.

Methods. We used the main hypothesis of V.Z. Vlasov concerning thin-walled bars. Solving motion equations analytically, we used the method of initial parameters and the known methods for differential equations solution. In the natural experiment we applied Autodesk Inventor system. In the course of the natural experiment on shaker, we used the method of smooth change in the frequency of sinusoidal vibrations.

Results. We provide the analytical solutions of the shapes and the natural frequencies of thin-walled bar with combined bisymmetrical section allowing for shears caused by bends and constrained torsion. We compared the results of the calculations conducted in CAE system and the results of the full-scale experiment to determine the frequencies of vibrations of box beam.

Conclusions. The results showed that the analytical calculations are in good compliance with the results of numerical and physical experiments and can be used for dynamic calculations of thin-walled structural elements, in particular, for the prevention of the destruction of structures in the event of resonance phenomena.
\end{abstract}

Keywords: thin-walled bar, free vibrations, warping, vibration frequency.

\section{Introduction}

Thin-walled bar structures are widely used in construction and mechanical engineering thanks to their low weight and low material capacity. Calculation method for this type of construction was formed in the twentieth century and later on it has been developing rapidly. Warping effect is a feature of thin bars calculation, which may be neglected in other cases. Currently, there are two approaches to this line of research: the first one takes into account the shears caused by constrained torsion, the second one does not. The first approach enables us to achieve quite high accuracy.

At the present time, finite element method is applied everywhere both for strength calculations, and for vibration calculations. For example, Tymoshenko beam vibrations of high amplitude were analyzed [1] using this method and considering transverse shear and rotational inertia in different boundary conditions. The results of numerical and experimental study of the natural frequencies of the thin-walled bar with open asymmetrical section considering shear strains and rotational inertia of the sections are presented in $[2,3]$. Work [4] presents the study of the natural frequencies of asymmetric homogeneous thin-walled bars. Work [5] analyzes the vibrations of thin-walled isotropic bars, highest local vibration modes and corresponding frequency. The research [6] studies the effect of length on the natural frequencies and forms of flat transverse vibrations of a thin inhomogeneous bar by means of numerical and analytical methods. The research [7] is devoted to the solution of differential equations of transverse vibrations of Timoshenko beam, which are used for thin-walled bars vibration analysis. Work [8] discusses flexural and torsional vibrations of thin-walled bar of open profile with one section symmetry axis. Static problem with the addition of inertia forces, i.e., using D'Alembert's principle, it is considered when solving the problem of bar vibration. The approach to the modeling of thin-walled plane grid system is implemented in the target computer programs.

In particular, restrained torsion shear strains are considered in the work of E.A. Beilin, which is devoted to stress-strain state [9]. V.I. Slivker proposed a semi-shear theory that takes into account a part of shear strains on the middle surface of bar walls caused by sectorial torque [10], which makes it possible to rather simplify analytical expressions.

Meanwhile, the use of analytical expressions to describe vibration shapes and frequency calculation, which is made quite seldom considering shear strains, allows us to speed up a design characteristics selection process, i.e. the process of the optimization of the structure [11]. In addition, the number of experimental studies on the dynamics of small thin-walled structures is quite small. Therefore, this study is very relevant. The purpose of this study was to establish analytical equations to calculate the frequencies and modes of vibrations of thinwalled bars with arbitrary section with a view of the effects of shear strains. The results were compared with the values obtained as a result of the modeling of vibrations in CAE system. In addition, we conduct natural experiment on the vibrations of box-section cantilever bar which resulted in the obtainment of values of natural vibration frequencies. 


\section{Materials and methods}

We used the main hypothesis for thin-walled bars proposed by V.Z. Vlasov [12], taking into consideration the shears caused by bends and constrained torsion. The method of initial parameters and known methods for the solution of differential equations were used in the establishment of equations.

Autodesk Inventor system was applied for numerical experiment.
Natural experiment was conducted on V8-440 HBT 900 CM8R shaker according to the method of smooth change in the frequency of sinusoidal vibrations according to GOST 30630.1.1-99 "Mechanical environment stability test methods for machines, instruments and other industrial products. Determination of dynamic characteristics of structure."

The bars with the rolled section made in accordance with GOST 8645-68 served as samples. St3ps steel served as the material (analogue to BS standard: 1449-27/23CR). Sensors dimensions and configurations are shown in Figure 1.
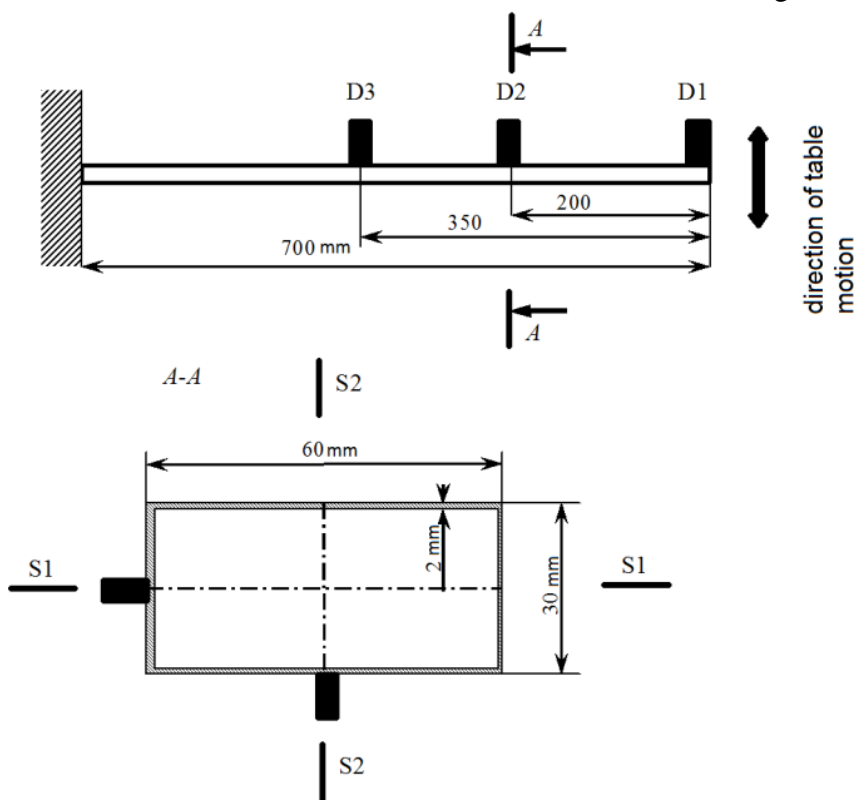

Figure 1. Bardimensions and sensor mounting places.D1, D2, D3 - sensors.S1, S2 - shaker table motion planes.

\section{Results}

Solution of motion equation of thin-walled bisymmetric profile bar.

$\rho J p \frac{\partial^{2} \theta(z, t)}{\partial t^{2}}-G J_{k} \frac{\partial^{2} \theta(z, t)}{\partial z^{2}}-\frac{G}{K_{33}}\left[\frac{\partial^{2} \theta(z, t)}{\partial z^{2}}-\frac{\partial \psi(z, t)}{\partial z}\right]=0$

$\rho J 3 \frac{\partial^{2} \psi(z, t)}{\partial t^{2}}-E I_{3} \frac{\partial^{2} \psi(z, t)}{\partial z^{2}}-\frac{G}{K_{33}}\left[\frac{\partial^{2} \theta(z, t)}{\partial z}-\partial \psi(z, t)\right]=0$

Flexural and torsional vibrations were considered separately upon receipt of the equations. The equation for free torsional and warping vibrations has the following form: where $t$ time; $Z$ - section coordinate; $\psi$ - warping function; $\theta$ - the angle of the twist of a section around a longitudinal axis; $\rho$ density of a bar material; $J_{3}$ - sectorial moment of inertia; $J_{p}$ - polar moment of inertia;

Here Roman numerals denote the derivative order with respect to $z$ and the following coefficients were determined:

In this equation, $A, B, C$ and $D$ are constants. These constants are selected based on edge conditions, i.e. on the bar ends
$J_{k}$ - geometrical factor of pure torsion stiffness; $k_{33}$ section shape coefficient.

The simplest periodic solution of the system equations (1) is so-called major vibrations in which the movement and angles vary in time harmonically. System solutions (1) for the torsional and warping vibrations were defined as $\theta(z, t)=\theta(z) \sin (v t+\alpha)$

$\psi(z, t)=\psi(z) \sin (v t+\alpha)$

where $\boldsymbol{V}$ - frequency of vibration; $\alpha$ - initial phase; $\theta(z)$ and $\psi(z)$ - law of distribution of the maximum (peak) deviations from a equilibrium position, these functions are the main vibration mode or natural mode. Following the substitution of (2) for (1), we established the differential equation for natural mode determination:

$$
\begin{aligned}
& \psi^{I}=\frac{\rho J p K_{33}}{G} v^{2} \theta+\left(J_{k} K_{33}+1\right) \theta^{I^{I}} \\
& \theta^{I V}+2 K_{31} \theta^{I I}-K_{32}=0
\end{aligned}
$$

The solution of the homogeneous differential equation $\theta(z)=e^{p_{3} z}$ was defined as $\theta(z)=e^{p_{3} z}$ where $p_{3}$ characteristic. Equation (3) has periodic solutions fixation conditions. Then, besides the function characterizing twist angle change, three more functions must be defined: 
warping characteristic - function, characteristics of bimoment and full torque moment, determined by the following formulas

After the introduction of ancillary quantities main vibration shapes for twist angle, warping functions, bimoment performance and full torque moment are finally presented in the form of:

$$
\left[\begin{array}{l}
\theta(z) \\
G \psi(z) \\
\frac{G B(z)}{E J_{3}} \\
\frac{M_{3}(z)}{\rho J_{p} v^{2}}
\end{array}\right]=\frac{1}{G(J k K 33+1)\left(P_{31}^{2}+P_{32}^{2}\right)}\left[\begin{array}{l}
A \\
A
\end{array}\right.
$$$$
\text { A2 }
$$$$
\text { A31 }
$$

The proposed approach was used to determine the frequency of all the vibration modes (flexural mode case is set out in [13]). These natural frequencies for the cantilever bar considered (see Figure 1) are shown in Table 1.

$\left.\begin{array}{ll}A 13 & A 14 \\ A 23 & A 24 \\ A 33 & A 34 \\ A 43 & A 44\end{array}\right]\left[\begin{array}{c}\theta 0 \\ G \psi 0 \\ \frac{G B(0)}{E J_{3}} \\ \frac{M_{3}(0)}{\rho J_{p} v^{2}}\end{array}\right]$

For comparison, the table shows the values of natural frequencies regardless of shifts.

Table 1. The natural frequencies at analytic calculation

\begin{tabular}{|c|l|c|c|c|}
\hline No. & \multicolumn{1}{|c|}{$\begin{array}{c}\text { Prevailing form of } \\
\text { vibrations }\end{array}$} & $\begin{array}{c}\text { Frequency, excluding } \\
\text { shifts caused by bend } \\
\text { and constrained } \\
\text { torsion, Hz }\end{array}$ & $\begin{array}{c}\text { Frequency allowing } \\
\text { for shifts caused by } \\
\text { bend and constrained } \\
\text { torsion, Hz }\end{array}$ & Difference, $\%$ \\
\hline 1 & Flexural in S2 No. 1 & 75.2 & 73.16 & 2.7 \\
\hline 2 & Flexural in S1 No. 1 & 130.4 & 127.25 & 2.4 \\
\hline 3 & Flexural in S2 No. 2 & 452.2 & 435.5 & 3.7 \\
\hline 4 & Flexural in S1 No. 2 & 767 & 740.14 & 3.5 \\
\hline 5 & Torsional No. 1 & 981 & 887.76 & 9.5 \\
\hline 6 & Flexural in S2 No. 3 & 1212 & 1147.2 & 5.3 \\
\hline 7 & Longitudinal & - & - & - \\
\hline 8 & Flexural in S1 No. 3 & 1998 & 1908 & 4.5 \\
\hline
\end{tabular}

\section{Results of numerical modeling}

We modeled the vibrations of a steel bar, the dimensions of which are shown in Figure 1. The presented results refer to a bar without sensors and to a bar with fixed sensors (in compliance with overall dimensions and mass of sensors).

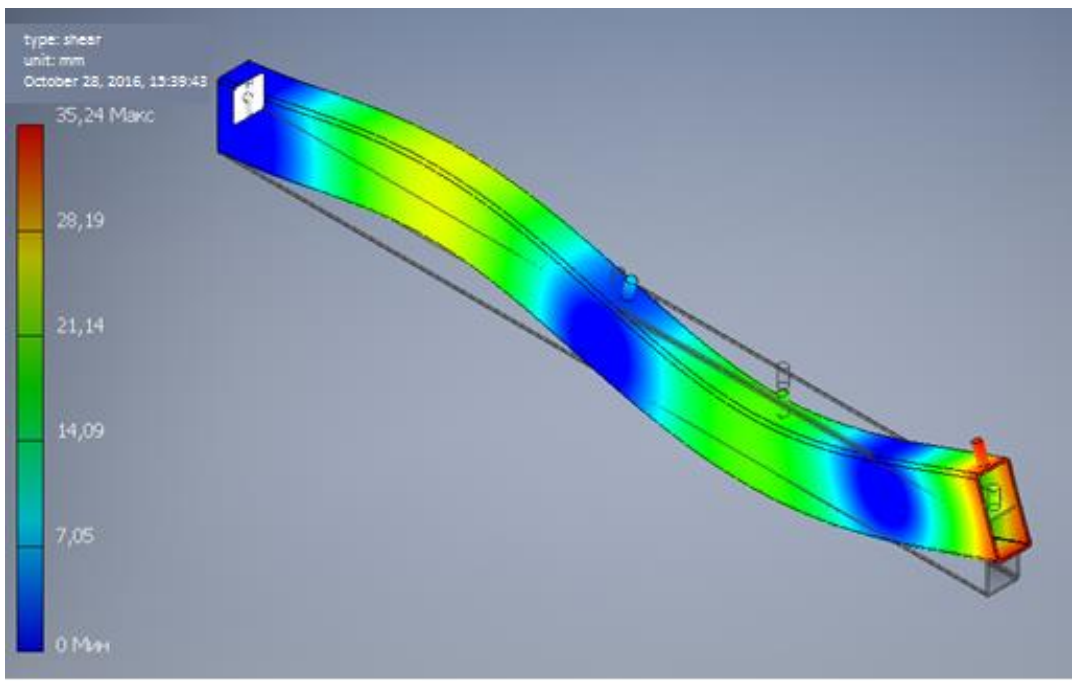




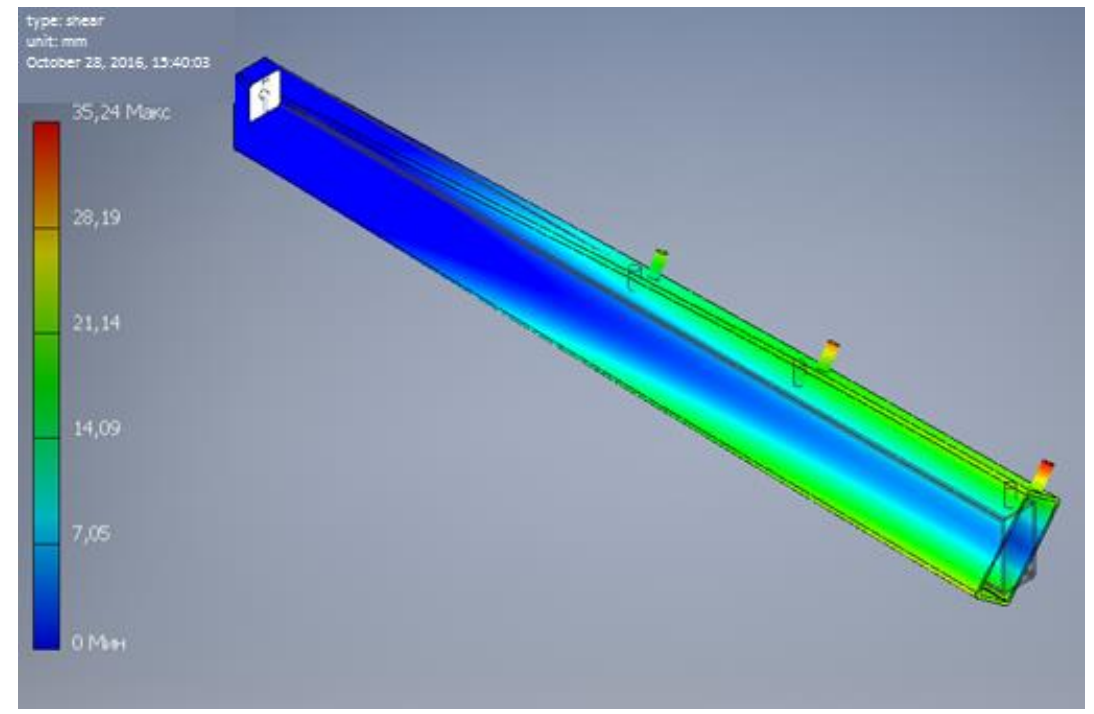

Fig. 2 Flexural and torsional forms obtained in Autodesk Inventor.

Table 2. Natural frequencies in numerical modeling

\begin{tabular}{|c|l|c|c|}
\hline No. & Predominant vibration mode & Frequency without sensors, $\mathrm{Hz}$ & Frequency with sensors, $\mathrm{Hz}$ \\
\hline 1 & Flexural in S2 No. 1 & 71.13 & 70.53 \\
\hline 2 & Flexural in S1 No. 1 & 123.07 & 121.96 \\
\hline 3 & Flexural in S2 No. 2 & 426.69 & 422.51 \\
\hline 4 & Flexural in S1 No. 2 & 726.97 & 720.67 \\
\hline 5 & Torsional No. 1 & 867.60 & 864,41 \\
\hline 6 & Flexural in S2 No. 3 & 1122.11 & 1106.16 \\
\hline 7 & Longitudinal & 1806.52 & 1793.00 \\
\hline 8 & Flexural in S1 No. 3 & 1874.33 & 1867.21 \\
\hline
\end{tabular}

\section{Natural experiment}

Figure 3 shows the mounting of samples with sensors on a vibrating table. Frequency changes caused by vibrations were in the range from $5 \ldots 37 \mathrm{~Hz}$ to $40 \ldots 2000 \mathrm{~Hz}$. Natural

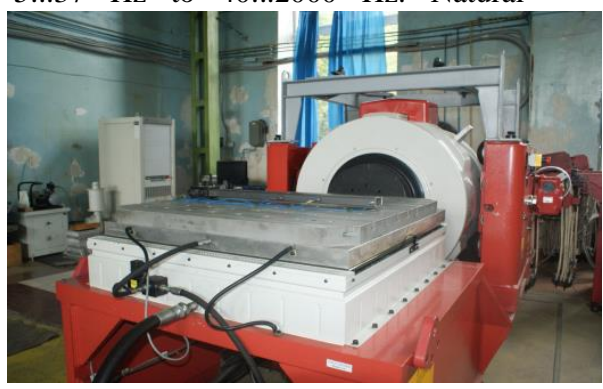

frequencies were determined according to the amplitude values of accelerations, that is shown in Figures 4 and 5.

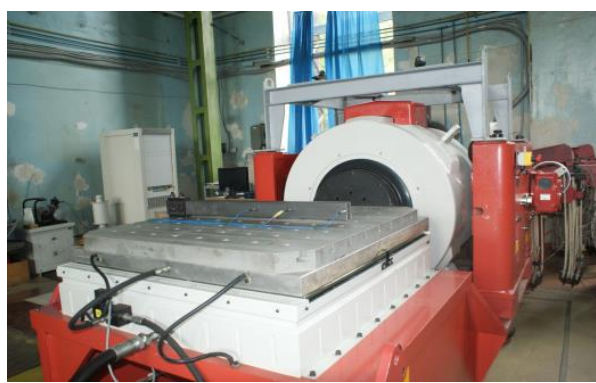

Figure 3. The samples mounted on a vibrating table. 


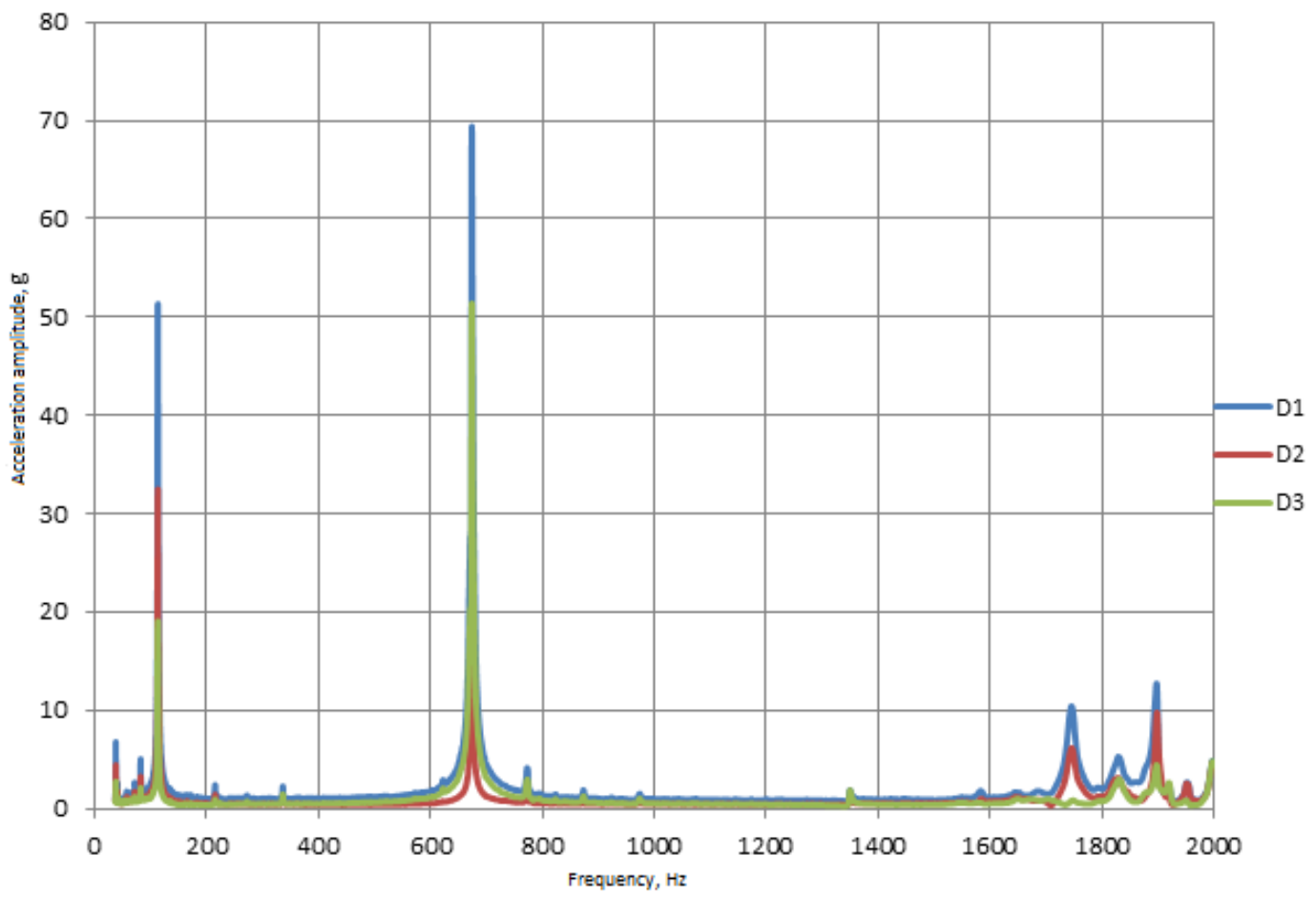

Figure 4.Accelerationamplitudes during vibrations in S1plane

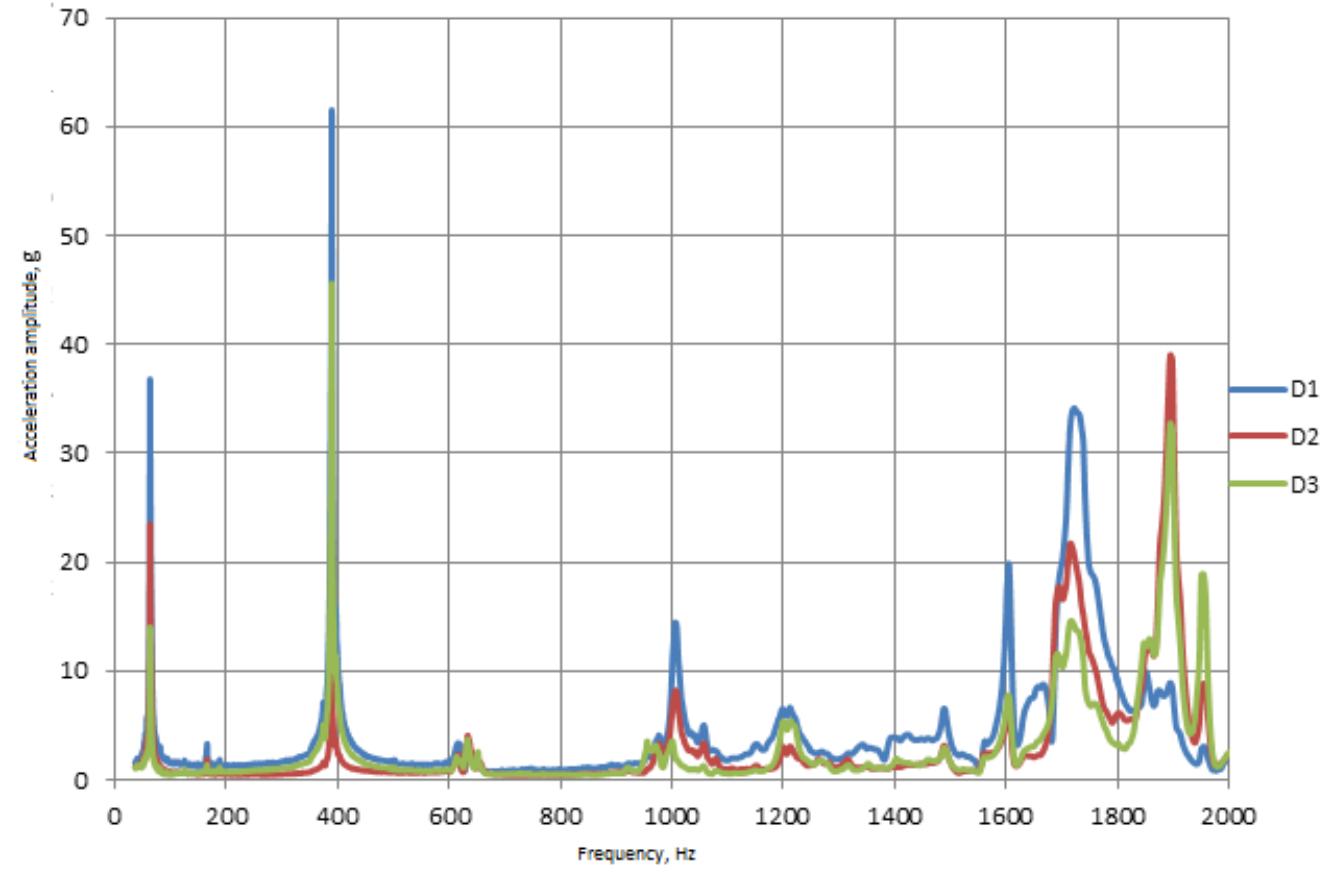

Figure 5.Accelerationamplitudes during vibrations in S2plane

Table 3. Natural frequencies determined by the results of the natural experiment

\begin{tabular}{|c|l|c|c|c|}
\hline No. & Prevailing vibration mode & Frequency, Hz & $\begin{array}{c}\text { Divergence from the } \\
\text { results of numerical } \\
\text { experiment (with } \\
\text { sensors), } \%\end{array}$ & $\begin{array}{c}\text { Divergence from the results } \\
\text { of analytical calculation, } \%\end{array}$ \\
\hline 1 & Flexural in S2 No.1 & 63.86 & 10.4 & 14.6 \\
\hline 2 & Flexural in S1 No.1 & 113.49 & 7.5 & 12.1 \\
\hline 3 & Flexural in S2 No.2 & 389.77 & 8.4 & 11.7 \\
\hline 4 & Flexural in S1 No.2 & 674.03 & 6.9 & 14.7 \\
\hline 5 & Torsional No.1 & 774.07 & 11.7 & 13.9 \\
\hline 6 & Flexural in S2 No.3 & 1007.08 & 9.8 & - \\
\hline 7 & Longitudinal & - & - & 9.3 \\
\hline 8 & Flexural in S1 No.3 & 1745.0 & 7.0 & 9.8 \\
\hline
\end{tabular}




\begin{tabular}{c} 
International Journal of Engineering \& Technology, $7(2.13)(2018) 7-12$ \\
International Journal of Engineering \& Technology \\
SPC \\
Website: $w$ ww.sciencepubco.com/index.php/IJET \\
Research Paper \\
\hline
\end{tabular}

\section{Discussion}

This version of the motion equations and their solution has a relatively simple form that allows to use them when searching for optimal solutions for bars and beams construction, for example, by the method described in [13].

Analysis of the results of analytical calculation shows that the record of shears caused by a bend and constrained torsion specifies the value of natural frequencies up to $5.3 \%$ for flexural vibrations and up to $9.5 \%$ for torsional vibrations. In this case, the frequency values are approximated to the results obtained by numerical modeling.

The main objective of the natural experiment was to determine bend frequencies. Thus, the results also allowed tracking the frequencies of torsional oscillations. This is due to some imperfection of the bar and torsional axial sensors shears. At high vibration frequencies in S2 plane, it becomes difficult to analyze acceleration amplitudes diagram since the vibrations frequencies of the walls of a bar get super imposed as a result of their low stiffness. In this case, the desired frequencies can be identified by determining shifts amplitudes for the corresponding mode shape.

Comparison of the results of the analytical and numerical calculation with the results of natural experiment revealed not only qualitative but also quantitative correspondence with small divergence: in the case of flexural shapes it didn't exceed $14.7 \%$.

\section{Conclusion}

We established the analytical expressions for the frequencies of natural flexural and torsional vibrations of thin-walled bar with bisymmetrical section having both open and closed areas, taking into account the shears caused by a bend and constrained torsion. They also make it possible to determine flexural vibration modes, angles of sectional twisting, warping function, bimoment, flexural moment, moments of pure and constrained torsion of a continuous bar taking into consideration secondary shears. The assessment of the effect of secondary shears on natural frequencies showed that this effect is significant and in the case of high vibration modes can amount up to $9.5 \%$ for the considered box-section bar. The comparison of the values of the frequencies obtained during the calculation according to the proposed method with the results obtained using the specialized bar modeling software systems based on volumetric finite elements, yielded the results that differed by $1-4 \%$, that enables us to use the proposed method. The divergence from the results of natural experiment did not exceed $14.7 \%$.

Thus, the proposed approach allows defining natural frequencies values more precisely and the results can be used for dynamic calculations and optimization of thin-walled structural elements, in particular, for the prevention of damage in the event of resonance phenomena.

This research was supported by the Government of the Orenburg region (Regional grant in the field of scientific and scientific-technical activities in 2017, agreement No.37).

\section{References}

[1] Gunda J. B., Gupta R. K., Janardhan G. R., Rao G. V. Large amplitude free vibration analysis of Timoshenko beams using a relatively simple finite element formulation / // International Journal of Mechanical Sciences. - 2010. - vol. 52. - no. 12. P. $1597-1604$.

[2] Ambrosini D. Experimental validation of free vibrations from nonsymmetrical thin walled beams // Engineering Structures. - 2010. - vol. 32. - no. 5. - P. 1324-1332.

[3] Ambrosini D. On free vibration of nonsymmetrical thinwalled beams // Thin-Walled Structures. - 2009. - vol. 47. no. 6-7. P. 629-636.

[4] Tanaka M., Bercin A. N. Free vibration solution for uniform beams of nonsymmetrical cross section using Mathematica // Computers and Structures. - 1999. - vol. 71. - no. 1. - P. $1-8$.

[5] Giunta G., Belouettar S. Higher-Order Hierarchical Models for the Free Vibration Analysis of Thin-Walled Beams // Hindawi Publishing Corporation Mathematical Problems in Engineering Volume. - $2015 . \quad$ Access: http://dx.doi.org/10.1155/2015/940347

[6] Akulenko L.D., NesterovS.V Anomalous dependence of the vibration frequencies of a rod in an elastic medium on the rod length // Mechanics of solids. -2010 . - vol. 45. - no. 2. P. 257-263.

[7] Attarnejad R., Semnani S.J., Shahba A. Basic displacement functions for free vibration analysis of non-prismatic Timoshenko beams // Finite Elements in Analysis and Design. - 2010. - vol. 46. - no. 10. P. 916-929.

[8] Chernov S. A. Thin-walled bar system dynamics tasks modeling // Software products and systems. - 2014. number 106. P. $171-176$.

[9] Beilin, E. A. Elements of the torsion theory of thin-walled bars of arbitrary profile [text] / E. A. Beilin. - SPSUACE Press- 2003. $113 \mathrm{p}$.

[10] Khosravi M., Mosaddeghi F., Oveisi, M., khodayari-b, A., Aerodynamic drag reduction of heavy vehicles using append devices by CFD analysis, Journal of Central South University, Volume 22, 2015, pp 4645-4652.

[11] Grebenyuk G. I., Gavrilov A. A., Yankov E. V. Calculation and optimization of continuous beam with thin-walled profile // Proceedings of the higher educational institutions. Building. - 2013. - number 7 (655) P. 3-11.

[12] Seyedhosseini, S. M., Esfahani, M. J., \& Ghaffari, M. A novel hybrid algorithm based on a harmony search and artificial bee colony for solving a portfolio optimization problem using a mean-semi variance approach. Journal of Central South University, 23(1), 2016, 181-188.

[13] Gavrilov A. A., Kudina L. I., Kucha G. V., Morozov N. A. Influence of geometrical characteristics of sections on the frequency values of the free flexural vibrations of thinwalled straight bars // Bulletin of Orenburg State University. - 2011. - number 5 (124) P. 146-150. 